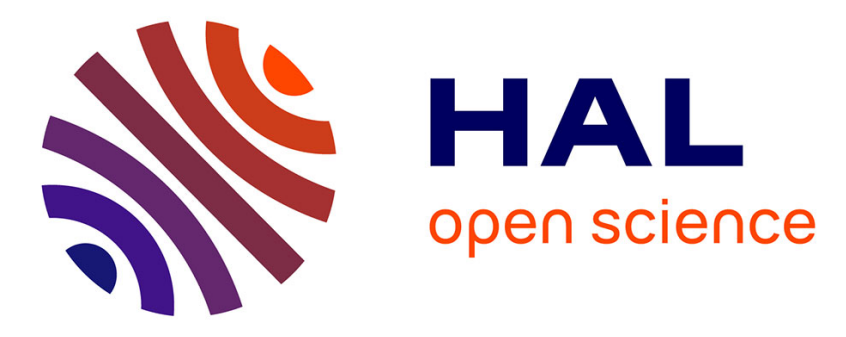

\title{
Innovative Brain-Computer Interface based on motor cortex activity to detect accidental awareness during general anesthesia
}

Sébastien Rimbert, Philippe Guerci, Nathalie Gayraud, Claude Meistelman, Laurent Bougrain

\section{To cite this version:}

Sébastien Rimbert, Philippe Guerci, Nathalie Gayraud, Claude Meistelman, Laurent Bougrain. Innovative Brain-Computer Interface based on motor cortex activity to detect accidental awareness during general anesthesia. IEEE SMC 2019 - IEEE International Conference on Systems, Man, and Cybernetics, Oct 2019, Bari, Italy. hal-02166934

\section{HAL Id: hal-02166934 \\ https://hal.inria.fr/hal-02166934}

Submitted on 27 Jun 2019

HAL is a multi-disciplinary open access archive for the deposit and dissemination of scientific research documents, whether they are published or not. The documents may come from teaching and research institutions in France or abroad, or from public or private research centers.
L'archive ouverte pluridisciplinaire HAL, est destinée au dépôt et à la diffusion de documents scientifiques de niveau recherche, publiés ou non, émanant des établissements d'enseignement et de recherche français ou étrangers, des laboratoires publics ou privés. 


\title{
Innovative Brain-Computer Interface based on motor cortex activity to detect accidental awareness during general anesthesia
}

\author{
Sébastien Rimbert ${ }^{1}$, Philippe Guerci ${ }^{2}$, Nathalie Gayraud ${ }^{3}$, Claude Meistelman ${ }^{2}$ and Laurent Bougrain ${ }^{1}$
}

\begin{abstract}
Accidental Awareness during General Anesthesia (AAGA) occurs in $1-2 \%$ of high-risk practice patients and is responsible for severe psychological trauma, termed posttraumatic stress disorder (PTSD). Currently, monitoring techniques have limited accuracy in predicting or detecting AAGA. Since the first reflex of a patient experiencing AAGA is to move, a passive Brain-Computer Interface (BCI) based on the detection of an intention of movement would be conceivable to alert the anesthetist and prevent this phenomenon. However, the way in which the propofol (an anesthetic drug commonly used for inducing and maintaining general anesthesia) affects the motor brain activity and is reflected by the electroencephalographic (EEG) signal has been poorly investigated and is not clearly understood. The goal of this forward-looking study is to investigate the motor activity behavior with step-wise increase of propofol doses in 4 healthy subjects and provide a proof of concept for such an innovative BCI.
\end{abstract}

\section{INTRODUCTION}

Brain-Computer Interfaces (BCIs) allow end users to interact with a system using modulations of brain activity which is partially observable in electroencephalographic (EEG) signals [1]. For several years, BCI progress has been focused essentially on rehabilitating people with severe neuromuscular disorders [2]. Nonetheless, the use of passive BCI to enhance surveillance of bioclinical signals is now emerging and could have a significant impact in the medical field [3].

During general anesthesia, the occurrence of one particular phenomenon is feared by both patients and anesthesiologists: Accidental Awareness during General Anesthesia (AAGA) [4]. This event can be defined as an unexpected awakening of the patient during a surgical procedure under general anesthesia [5]. This situation occurs when the depth of anesthesia is insufficient to compensate for the surgical and environmental stimuli [6], [7]. In high-risk practices, the AAGA rate is up to $1 \%$ [8], [9] which is considered as high, and is especially difficult to detect when patients are paralyzed by the use of neuromuscular blocking agents.

Following this distressing experience, patients may be affected by post-traumatic stress disorder (PTSD), which can have a severe impact on the victim's life [10], [6]. Additionally, the occurrence of such an event may also generate anxiety in anesthesiologists [9]. Finally, it is in the top 3 causes of anesthesia closed claims which induce

\footnotetext{
*This work was not supported by any organization

${ }^{1}$ Sébastien Rimbert and Laurent Bougrain are with the Université de Lorraine, Inria, LORIA, F-54000 Nancy, France sebastien.rimbert@inria.fr

${ }^{2}$ Nathalie Gayraud is with Université Côte d'Azur, Inria, Sophia-Antipolis Méditerrannée, Athena team, France

${ }^{3}$ Philippe Guerci and Claude Meistelman are anesthesiologists at the University Hospital of Nancy, France
}

additional costs for the hospital [11]. These observations highlight the importance of detecting and preventing this phenomenon.

There are currently two ways to monitor the depth of anesthesia: observing clinical features (e.g., heart rate, blood pressure, movement, sweating) or using EEG analysis, mainly of the frontal cortex activity. Unfortunately, an anesthesiologist's observation of clinical signs is not accurate enough to prevent AAGA during a surgery [12]. Indeed, clinical signs can only be an indirect monitoring of the cerebral state of patients and cannot always predict AAGA before it occurs. New indexes using part of the EEG signal recorded over the frontal lobe, such as the Bispectral Index (BIS), the Patient State Index (PSI) or the Entropy [13] try to define a patient's depth of anesthesia. Although these devices are already in use [14], existing studies have failed to demonstrate the superiority of these monitors compared to clinical surveillance or end-tidal anesthetic gas for the prevention of AAGA [15]. Some studies have actually shown the unreliability of these techniques [16], highlighting that no technique is satisfactory in evaluating the depth of general anesthesia and detecting intraoperative awakening.

'I couldn't breathe, couldn't move or open my eyes, or tell the doctor that I wasn't asleep'. This kind of testimony underlines that, during AAGA, a patient's first reaction is having the willing to move so as to alert the medical staff of this terrifying situation. However, to enhance/ease surgery, many procedures require the use of paralytics, which causes a neuromuscular relaxation and inhibits any movement [4]. The following question arises: How to detect an intention to move to alert the anesthesiologist when the patient is experiencing AAGA?

Nowadays, an attempted movement or a Motor Imagery (MI) can be detected by extracting sensorimotor patterns from the EEG signal [17]. These sensorimotor rhythms are characterized i) before and during an imagined movement, by a gradual decrease of power in -mainly- the mu/alpha (7$13 \mathrm{~Hz})$ and beta $(15-30 \mathrm{~Hz})$ bands and ii) after the end of the motor imagery, by an increase of power in the beta band. These modulations are respectively known as Event-Related Desynchronization (ERD) and Event-Related Synchronization (ERS) or post-movement beta rebound [18], [19]. Hence, to detect the occurrence of AAGA during surgery, we suggest to analyze the ERD and ERS modulations and design a new passive BCI. Such a BCI would identify the occurrence of AAGA by detecting an intention of movement from the patient, using the associated motor patterns recorded in EEG signals. 
Nevertheless, detecting MI, especially asynchronously, is not a trivial task for current MI-based BCI. To answer this issue, we showed in a previous study that a BCI based on the median nerve stimulation (MNS) is a promising approach to detect AAGA without any trigger and with a higher performance [20]. Indeed, our results indicated that a MI significantly modulates the ERDs and ERSs previously generated by a MNS and also that classification based on MNS is more efficient than conventional classification based on MI versus rest. However, this work was carried out on subjects without propofol administration and needs to be assessed under anaesthesia conditions. There is little information concerning the effect of propofol on the ERD and ERS modulations. One such study was conducted by Blockland et al., who examined the effect of propofol on the EEG signals of the motor cortex and verified the relevance of this approach for improved monitoring of AAGA [21].

In the present study, we sought to evaluate if a BCI based on MNS can be used to detect a MI under light anaesthesia conditions, i.e. under propofol. To investigate this issue, 4 right-handed healthy subjects performed 3 motor tasks at 3 propofol concentrations. We analyze the ERD and ERS and compare the BCI accuracy for all concentrations. Our results suggest that the anesthetic state does not significantly modulate ERD and ERS patterns. Moreover, it allows MI detection by a BCI. Finally, we show that a BCI based on median nerve stimulation seems to be more efficient than conventional classification based on MI versus rest and could be used to prevent intraoperative awareness.

\section{MATERIALS AND METHODS}

\section{A. Participants}

Four right-handed healthy volunteers (only males; from 19 to 25 years-old) were recruited for this preliminary study. All voluntary subjects satisfied the inclusion criteria: male, age between 18 and 28 years old, body mass index between 22 and $28 \mathrm{~kg} . \mathrm{m}^{-2}$, right-handed, be affiliated to a social security, and without any medical history which could have influenced the task, such as diabetes, antidepressant treatment, neurological disorders or history of drug or alcohol abuse.

The experiment was held in an approved location by the French Regional Agency for Health ( $\left.{ }^{\circ} 2017-2500\right)$, in the surgical intensive care unit JM. Picard, Department of Anesthesiology and Critical Care Medecine, at the University hospital of Nancy-Brabois, France. The study was conducted in accordance with the principles of the Declaration of Helsinki and the Medical Research Involving Human Subjects Act. This study has been approved by a national ethical committee (Comit de Protection des Personnes Ile de France 1). The experiment has also been approved by the French Agency for the Safety of Health Products $\left(\mathrm{N}^{\circ}\right.$ EUDRACT 2017-004198-15). Finally, the study protocol was registered on clinicaltrials.gov (NCT03362775).

\section{B. Experimental Tasks}

The aim of this research was to investigate the occurrence of motor patterns for 4 different motor tasks: real movement (RM), motor imagery (MI), median nerve stimulation during rest (MNS) and median nerve stimulation during motor imagery (MI + MNS) under 3 propofol concentrations at the effect site: $0 \mu \mathrm{g} \cdot \mathrm{ml}^{-1}, 0.5 \mu \mathrm{g} \cdot \mathrm{ml}^{-1}$, and $1 \mu \mathrm{g} \cdot \mathrm{ml}^{-1}$. These three concentrations have been wisely chosen considering the literature on this topic [21] and the concentrations usually used to induce general anesthesia (i.e. i2 $\mu \mathrm{g} \cdot \mathrm{ml}^{-1}$ ). In particular, these low to intermediate concentrations were chosen to ensure that the voluntary subject was not unconscious and could still perform the motor tasks.

The order of the 4 motor tasks was randomized for each subject in order to avoid fatigue, gel drying or other confounding factors that might have caused possible biases in the results. For each task, the subject had to perform the task 52 times in one run. At the beginning of each run, the subject remained relaxed for 15 seconds. Subjects were asked to keep their eyes closed as during a general anesthesia. We have previously shown that this condition causes minimal disruption in term of ERDs and ERSs during motor tasks [22].

1) Motor imagery: For the MI condition (C1), subjects had to imagine an isometric grasp between the thumb and the index finger on a pointer button, i.e. they had to try to feel a maximum of sensations caused by the real movement but without actually performing any movement. A low frequency beep indicated when the subject had to start the MI. The grasping MI was maintained during 2 seconds then a second beep indicated the end of the imagined task.

2) Median nerve stimulation only: The Median Nerve Stimulation (MNS) only condition (C2) consisted of a series of electric stimulation of the median nerve during rest. We placed the two electrodes of stimulation on the wrist according to the standards as in [23], [24]. We considered as an inclusion criterion for our population of subjects the fact that the stimulation was not felt as painful and that it caused a slight movement between the thumb and the index finger of the voluntary subject. The stimulation intensity was adapted for each subject and varied between 8 and $15 \mathrm{~mA}$.

3) Motor imagery with median nerve stimulation: During the MI + MNS condition (C3), subjects had to perform a motor imagination during 2 seconds. Additionally, an MNS occurred at 750 milliseconds after the first beep. Consistently with the previous conditions, a low frequency beep indicated when the subject had to start the MI and a high frequency beep indicated when he had to stop it. We chose the 750 milliseconds of delay according to the reaction time of an average person, in order to stimulate during the ERD corresponding to the start of the imagination. A previous study confirmed that a MNS during the MI would not inhibit the ERD produced by this MI [20].

\section{Experimental Design}

All participants were interviewed by the medical investigator before starting the study to assess compliance with the 
inclusion/exclusion criteria.

Three days before the experiment, an appointment was made at the INRIA center in Nancy to (i) inform the subject about the experimental conditions (e.g. motor tasks, sound cues, duration for each task), (ii) learn the kinesthetic motor imagery task, (iii) select the MNS intensity needed to produce a micro movement between the thumb and the index finger and (iv) to present to the subjects the material used for the research.

On the day of the experiment, the voluntary subject took part to one session of 180 minutes divided in 3 phases: 1) installation of the EEG cap (30 minutes) and anesthesia equipment; 2) execution of the tasks under 3 propofol concentrations at the effect site $\left(0 \mu \mathrm{g} . \mathrm{ml}^{-1}, 0.5 \mathrm{~g} \cdot \mathrm{ml}^{-1}\right.$, and 1 $\mu \mathrm{g} . \mathrm{ml}^{-1}$ ) (90 minutes) ; 3) EEG cap removal, debriefing and completion of two surveys of street abilities [25] to assess the subject's ability to leave the hospital safely (40 minutes).

In addition to the EEG cap, all subjects were asked to rest in a semi recumbent $\left(15^{\circ}\right)$ supine position and were continuously monitored with an electrocardiogram (ECG), a non-invasive blood pressure measurement (NIBP), a pulse oximetry $\left(\mathrm{SpO}_{2}\right)$ (GE Healthcare) and an oxygen supplement delivered by nasal cannula $\left(21 . \mathrm{min}^{-1}\right)$. A $24 \mathrm{G}$ peripheral catheter (BD Insyte Autogard) were inserted in the left forearm and continuously infused with a crystalloid solution (Isofundine, B-Braun). Finally, the subjects were infused with propofol LIPURO $1 \%\left(10 \mathrm{mg} \cdot \mathrm{ml}^{-1}\right.$, B Braun, Melsungen) using a target-controlled infusion pump with Schnider pharmacokinetic model (B-Braun Perfusor, B Braun, Melsungen) at the effect site. During the first session of the experiment, no infusion of propofol was performed ( 0 $\left.\mu \mathrm{g} . \mathrm{ml}^{-1}\right)$.

\section{Data Acquisition}

EEG signals were acquired at $2048 \mathrm{~Hz}$ using the OpenViBE platform with a Biosemi Active Two 128-channel EEG system, arranged in the Biosemi's ABC system covering the entire scalp. An external electromyogram (EMG) electrode was added in order to verify that there was no movement during the MI task.

\section{E. Data Pre-Processing}

Offline analyses were performed using the EEGLAB toolbox [26] on Matlab2016a (The MathWorks Inc. Natick, MA, USA) and the MNE, Pyriemann and Scikitlearn Python libraries. Considering the large number of electrodes used in this study (eg. 128) and the purpose of this research (motor patterns over the motor cortex) we chose to use a common average referencing (CAR) performed using EEGLAB [27].

\section{F. Event-related Spectral Perturbations}

1) Time-Frequency Analysis: To analyze the differences between propofol concentrations, EEG signals were resampled at $128 \mathrm{~Hz}$ and divided into 9-second epochs corresponding to 2 seconds before and 7 seconds after the motor task for each run. We then performed an event-related spectral perturbation (ERSP) between 8-30 Hz. We used a 256 point sliding fast Fourier transform (FFT) window with a padratio of 4 . We computed the mean ERSP from $1 \mathrm{~s}$ before the task up to $7 \mathrm{~s}$ after the task (Fig. 1A). ERSP allows to visualize event-related changes in the averaged power spectrum relative to a $2 \mathrm{~s}$ baseline interval, taken $1 \mathrm{~s}$ before each trial.

2) Topographic Map: Brain topography allows us to localize which part of the brain was involved when the subject performed the requested task. More precisely, it allows us to understand how MI + MNS and MNS conditions can be discriminated during the $[-0.5,1.5] \mathrm{s}$ interval. We decided to compute ERSPs in a merged band (mu+beta, 8-30 Hz) for MNS and MI+MNS conditions (Fig. 1B).

\section{G. Classification}

We considered two classification tasks: MI vs Rest, and MI + MNS vs MNS. For each classification, we computed the accuracy corresponding to each propofol concentration. During a general anesthesia, subjects are laying on the back of their heads, which makes it a challenging task to have good signals from electrodes everywhere on the scalp. Therefore, for this experiment, we only considered the electrodes that were located on the motor cortex $(n=48)$, which are those that are the most pertinent for imagined movements. Each EEG signal was then resampled at $256 \mathrm{~Hz}$ and band-passed in a $8-30 \mathrm{~Hz}$ filter with a 4th order zerophase Butterworth filter.

For the MI condition, each trial was segmented in two parts: the MI segment starts 0.5 second after the go signal (1st beep) and lasts $1.5 \mathrm{~s}$ while the rest segment starts 3 seconds after the stop signal (2nd beep) and lasts $2 \mathrm{~s}$. For $\mathrm{MI}+\mathrm{MNS}$ and MNS conditions, we selected a time window of $2 \mathrm{~s}$ starting $0.5 \mathrm{~s}$ before the median nerve stimulation for all trials of both conditions.

We collected 52 trials for each class, which resulted into 104 trials in total for both classes. In the 5-fold cross validation scheme, for each fold, 84 trials (42 per class) were used to train the classifier and the 20 remaining trials (10 per class) were used to validate it. We used a Linear Discriminant Analysis (LDA) trained and evaluated using Common Spatial Pattern (CSP) features generated from the first and last 4 CSP filters [28] (referred to as CSP+LDA). The CSP method is widely used in the field of MI-based BCI, as it provides a feature projection onto a lower dimensional space that minimizes the variance of one class while maximizing the variance of the other.

Once selected filters $W$ were obtained from band-pass EEG signals $X$, a CSP feature vector $f$ can be computed as follows [29]:

$$
\begin{gathered}
f=\log \left(W X X^{\top} W^{\top}\right)=\log \left(W C W^{\top}\right)=\log (\operatorname{var}(W X)) \\
\text { III. RESULTS }
\end{gathered}
$$

\section{RESULTS}

\section{A. Impact of the Propofol concentration on ERSPS}

To investigate how ERSPs are modulated according to the propofol concentration, we perform a time frequency analysis and display topographic maps. 


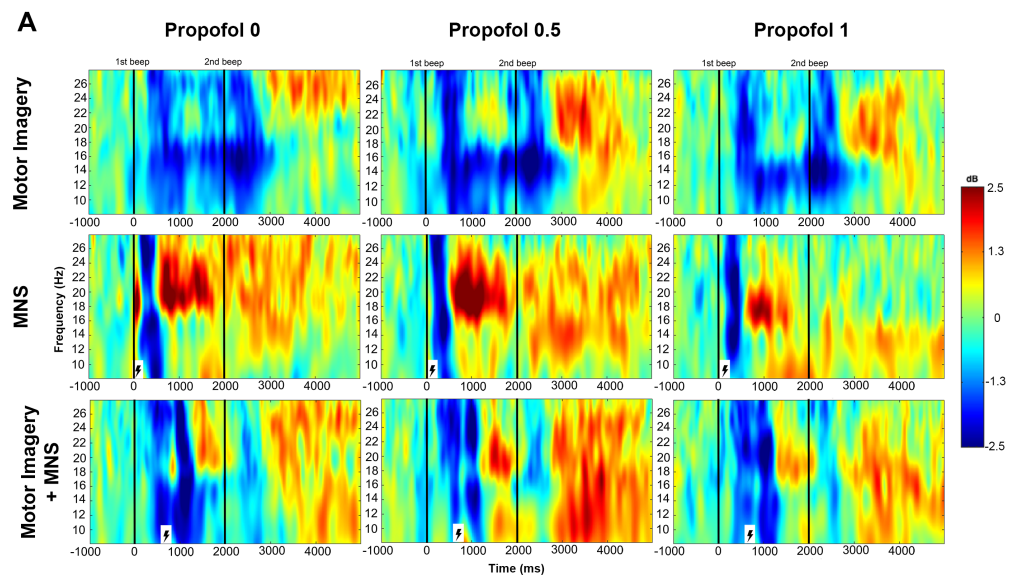

Fig. 1. A. Time-frequency grand average analysis (ERSP) for MI, MNS and MI+MNS motor tasks under 3 propofol concentrations $\left(0 \mu\right.$ g.ml ${ }^{-1}, 0.5$ $\mu \mathrm{g} . \mathrm{ml}^{-1}$, and $1 \mu \mathrm{g} \cdot \mathrm{ml}^{-1}$ ) for electrode $C_{3}$. Black lines indicate when the motor task started and finished. Red color corresponds to a strong ERS and blue to a strong ERD. B. Topographic map of ERSPS grand average in the alpha/mu+beta band $(8-30 \mathrm{~Hz})$ during two conditions: MI + MNS and MNS only for the [-0.5-1.5] s interval. A red colour corresponds to a strong ERS and a blue one to a strong ERD.

1) Time-Frequency analysis: Time-frequency maps are used to show the EEG modulation over the motor cortex and are useful for establishing the frequency and time windows in which ERSP appears for the electrode C3 (Fig. 1A).

Without propofol, for the MI task, the results shown an ERD during the MI task in the mu $(7-13 \mathrm{~Hz})$ and the beta $(18-30 \mathrm{~Hz})$ frequency bands (Fig. 1A). This ERD started $400 \mathrm{~ms}$ after the beep sound. This delay corresponds to the subject's reaction time, and was maintained until $2800 \mathrm{~ms}$. Then, an ERS was present in the high beta band (22-30 $\mathrm{Hz}$ ). At the concentrations of propofol $0.5 \mu \mathrm{g} \cdot \mathrm{ml}^{-1}$ and 1 $\mu \mathrm{g} \cdot \mathrm{ml}^{-1}$, very few changes were observable compared to the condition without propofol. Indeed, the ERD phase was very similar while the ERS was more effective in the medium beta $(16-24 \mathrm{~Hz})$ band.

For the MNS condition, no significant change can be observed in the different concentrations of propofol. After every MNS, a strong ERD appears in the mu+beta band, followed by a stronger ERS in the beta band. The beta rebound seems to be less sustained as the concentration of propofol increases.

If the median nerve is stimulated during a MI then a continuous ERD appears and is longer than for a MNS only. However, no change occurs if the dose of propofol is raised.

2) Topographic map: Analysis of these time-frequency maps showed that both mu $(7-13 \mathrm{~Hz})$ and beta $(18-30 \mathrm{~Hz})$ bands were impacted in term of ERSPSs in all three conditions. Since the previous results for the MI are consistent with the literature and the purpose of this study is to verify that we can discriminate the MNS and the MNS+MI conditions, we will only look into the these conditions.

In accordance with Fig. 1A, we have shown the topographic figures of the ERDs/ERSs in the time interval [$0.5-1.5$ ] s, corresponding to the period during which both MNS and MNS+MI states appeared to be the most distinct. The results showed that a classification based on MNS vs MNS+MI can be performed during this period of time. Indeed, for the MNS task, the modulations were very weak, which was explained by an ERD and an ERS expressed in the same time interval, while for the MNS+MI task, ERD was strongly expressed over the motor cortex area.

Propofol sedation did not change the difference between MNS and MNS+MI although the increase in propofol concentration tends to contralateralise the ERD for this task.

\section{B. Effect of the Propofol concentration on the classification accuracy}

To assess the potential impact of propofol on the performance of a BCI, we computed the classification accuracy of each each motor task for each propofol concentration and subject in a 5-fold cross validation scheme. Figures 2 and 2 represent the results for each classification task in the form of bar charts. Each bar represents the average classification accuracy obtained over each one of the 5 folds. The standard deviation is also displayed, as well as the average accuracy over all subjects for each propofol concentration.

1) MI vs Rest: The offline analysis results regarding the discrimination between a Motor Imagery and a Rest condition under different propofol concentrations are presented on figure 2. Each bar corresponds to the average classification accuracy of a single subject, for each one of the three sessions where different propofol concentrations were administered. The average classification accuracy obtained from subjects 2 and 3 increase when propofol concentration increases. Overall, propofol does not appear to hinder performance. For the baseline condition, i.e. no propofol (Propofol 0 ), the average classification performance across subjects was $74 \%$ while for 0.5 and $1 \mu \mathrm{g} \cdot \mathrm{ml}^{-1}$ the average classification accuracy increases to $78 \%$ and $84 \%$ respectively.

2) $M N S+M I$ vs MNS: Figure 2 shows the results for the MNS+MI vs MNS classification task. When no propofol is administered, the average classification accuracy is $81.5 \%$. The accuracy increases when $0.5 \mu \mathrm{g} \cdot \mathrm{ml}^{-1}$ of propofol is administered, reaching $88.5 \%$. For $1 \mu \mathrm{g} \cdot \mathrm{ml}^{-1}$ of propofol, the average classification accuracy decreases to $81.5 \%$. If we observe each subject individually, we see that propofol 

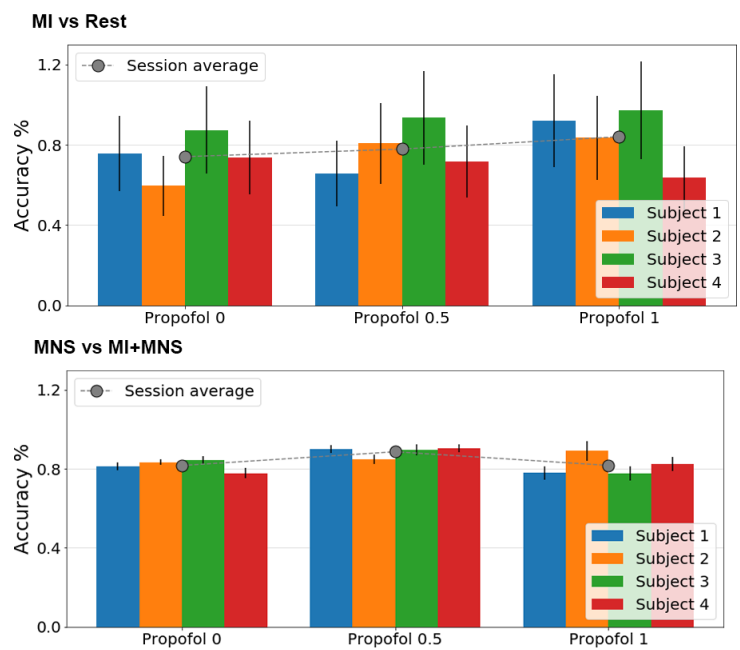

Fig. 2. Bar chart showing the distribution of classification accuracy (4 subjects) for MI vs Rest and MNS vs MI+MNS under 3 propofol concentrations $\left(0 \mu \mathrm{g} \cdot \mathrm{ml}^{-1}, 0.5 \mathrm{~g} \cdot \mathrm{ml}^{-1}\right.$, and $\left.1 \mu \mathrm{g} \cdot \mathrm{ml}^{-1}\right)$. The session average is displayed in grey.

administration either has a positive effect on classification accuracy (MI vs Rest) or has no effect at all.

Compared to the previous MI vs Rest classification task, we observe that the classification accuracy is on average higher for the first two propofol concentrations $(0$ and 0.5 $\mu \mathrm{g} . \mathrm{ml}^{-1}$ ). These results are statistically significant when performing a Welch's t-test, where the p-values are ; 0.05 for both concentrations. The performance differences between the two classification tasks is not statistically significant for the third propofol concentration (Propofol 1). The results are also less variable for each cross validation scheme, which implies that the noise levels are lower for the MNS+MI classification task.

\section{DISCUSSION}

In this study, we aimed to provide proof of concept for a solution towards reducing the intraoperative awareness occurrences during general anaesthesia. This kind of awakening during surgery can generate PTSDs and no reliable tool exists today to prevent its occurrence. Because patients undergoing AAGA have the reflex to move to warn the medical staff, we believe that a passive BCI based on motor patterns will help detect AAGA.

\section{A. ERD/ERS modulations during a propofol sedation}

Analysing the effect of propofol on ERD and ERS modulations over the motor cortex is critical to the design of a new passive BCI for AAGA detection. It is possible that sedation deactivates the motor cortex or significantly dampens the patterns that are typically detected during a motor imagery. Should this occur, it would imply that detecting those patterns would be impossible. Although very few studies have investigated the propofol effect on the EEG signal over the motor cortex, Blokland et al have previously shown a decrease of the ERD phase for similar concentrations $(0.75$ $\mu \mathrm{g} . \mathrm{ml}^{-1}$ and $1.5 \mu \mathrm{g} \cdot \mathrm{ml}^{-1}$ ) but with a very high inter-subject variability [21]. Our results are in contradiction with this finding. For the 3 motor tasks (MI, MNS and MI+MNS), we observe few changes in terms of ERDs and ERSs patterns [30]. In order to validate these findings, more subjects should be recorded and the baseline used for each propofol session should be validated as unchanged. This would allow a better understanding of the effect of sedation on ERD and ERS patterns.

\section{B. Does propofol improves BCI performance?}

While our results show that the ERD and ERS remain relatively unchanged for electrode $\mathrm{C} 3$ (fig. 1A), classification accuracy was investigated separately. Our results clearly show that, for both classification tasks, propofol either increases classification accuracy or has no effect on it (fig. 2).

Explaining why propofol increases performance in some cases is a delicate matter. We attempt to formulate a hypothesis based on the testimonial of our subjects: all four subjects described their state of mind while being injected with propofol as "focused". At the same time, they all declared that they felt "time was moving faster". In fact, many authors have underlined the significance of being calm and relaxed and its effects on oscillations and BCI performances. Nevertheless, to the best of our knowledge, this has never been experimentally tested with anesthetics. It is therefore possible that being lightly sedated indirectly influences the variability of the Rest condition, as subjects are more relaxed and focused on the movement task. This would justify our findings, especially for the intermediary condition (Propofol $0.5)$ where the subjects are still concentrated enough to perform the motor task.

\section{Median Nerve Stimulation as an innovative trigger for intraoperative awareness detection}

Our results for the MI vs Rest task encourage us to conceive the design of a passive MI-based BCI to detect accidental awareness during general anesthesia. Nevertheless, movement detection in such a BCI cannot be based on an external stimulus. Indeed, the patient might not recognize such a stimulus if they are in a state of anesthesia. Asynchronous $\mathrm{BCI}$ are however less performing, since the absence of a trigger induces more uncertainty.

Stimulating the median nerve can be seen as a solution to this issue. Many studies have shown that stimulating the median nerve generates ERD and ERS patterns [31], [32], [23]. Moreover, these patterns are furthermore modulated if the stimulation takes place during a motor task. In a previous study, we confirmed these results in the zero propofol condition and thus demonstrated the MNS+MI vs MNS classification task yields high performances [20]. This study enhances the importance of our findings, since it validates that these performances can also be obtained under propofol influence. In light of the results of this study, it is clear that using median nerve stimulation is a satisfactory solution to the trigger absence issue although some studies have suggested that the median nerve stimulation might hasten awakening [33]. 


\section{CONCLUSIONS}

The main objective of this study was to investigate how the EEG signal of the motor cortex was modulated with increasing sedation of propofol. Results indicated few variations in terms of ERDs and ERSs for each motor task, suggesting that intention to move can be detected under propofol. The second goal was to verify that a passive BCI could detect the intention of movement, even when the subject is under propofol. Our results confirm that a state-of-the-art BCI can discriminate MI vs Rest under propofol. Indeed, classification accuracies are better for 3 out of 4 subjects, highlighting the positive impact of propofol-induced relaxation on good performance [34]. However, in real conditions, this kind of classification will be complicated to perform, as no sound cue will be delivered to inform the subject when to start and stop an intention to move. To solve this problem, we proposed to use a median nerve stimulation as a routine procedure and classify MNS vs MNS+MI to detect an intention of movement during a general anesthesia. Our results showed that the classification rate is not impacted by propofol sedation, highlighting that this technique could be used to detect accidental awareness during general anesthesia.

\section{REFERENCES}

[1] E. W. W. Jonathan Wolpaw, Ed., Brain-Computer Interfaces: Principles and Practice. Oxford university press, 2012.

[2] I. Lazarou, S. Nikolopoulos, P. C. Petrantonakis, I. Kompatsiaris, and M. Tsolaki, "Eeg-based braincomputer interfaces for communication and rehabilitation of people with motor impairment: A novel approach of the 21st century," Frontiers in Human Neuroscience, vol. 12, p. 14 2018.

[3] P. Aricò, G. Borghini, G. D. Flumeri, N. Sciaraffa, and F. Babiloni, "Passive BCI beyond the lab: current trends and future directions," Physiological Measurement, vol. 39, no. 8, p. 08TR02, aug 2018.

[4] S. Tasbighou, M. Vogels, and A. Absalom, "Accidental awareness during general anaesthesia - a narrative review," Anaesthesia, vol. 73, no. 1, pp. 112-122, 2018.

[5] D. Almeida, "Awake and unable to move: what can perioperative practitioners do to avoid accidental awareness under general anesthesia?" J Perioper Pract, vol. 25, no. 12, pp. 257-261, 2015.

[6] K. MacGregor, "A waking nightmare: how can we avoid accidental awareness during general anaesthesia?" J Perioper Pract, vol. 23, no. 9, pp. 185-90, 2013.

[7] P. Myles, K. Leslie, J. McNeil, A. Forbes, and M. Chan, "Bispectral index monitoring to prevent awareness during anaesthesia: the b-aware randomised controlled trial," The Lancet, vol. 363, no. 9423, pp. 1757 $-1763,2004$.

[8] P. Sebel, T. Bowdle, M. Ghoneim, I. Rampil, R. Padilla, T. Gan, and K. Domino, "The incidence of awareness during anesthesia: a multicenter united states study." Anesth Analg, vol. 99, no. 3, pp. 8339, 2004.

[9] L. Xu, A.-S. Wu, and Y. Yue, "The incidence of intra-operative awareness during general anesthesia in china: a multi-center observational study," Acta Anaesthesiologica Scandinavica, vol. 53, no. 7, pp. 873$882,2009$.

[10] J. E. Osterman, J. Hopper, W. J. Heran, T. M. Keane, and B. A. van der Kolk, "Awareness under anesthesia and the development of posttraumatic stress disorder," General Hospital Psychiatry, vol. 23, no. 4, pp. 198 - 204, 2001.

[11] R. Mihai, S. Scott, and T. Cook, "Litigation related to inadequate anaesthesia: an analysis of claims against the NHS in england 19952007." Anaesthesia, vol. 64, no. 8, pp. 829-35, 2009.

[12] P. A. Punjasawadwong, Y and N. Bunchungmongkol, "Bispectral index for improving anaesthetic delivery and postoperative recovery," Cochrane Database of Systematic Reviews, no. 6, 2014.

[13] C. Kent and K. Domino, "Depth of anesthesia," Curr opin Anaesthesiol, vol. 22, no. 6, pp. 782-787, 2009.
[14] Y. Punjasawadwong, A. Phongchiewboon, and N. Bunchungmongkoi, "Bispectral index for improving anaesthetic delivery and postoperative recovery," Cochrane Database Syst Rev, vol. 17, no. 6, 2014

[15] M. S. Avidan, L. Zhang, B. A. Burnside, K. J. Finkel, A. C. Searleman, J. A. Selvidge, L. Saager, M. S. Turner, S. Rao, M. Bottros, C. Hantler, E. Jacobsohn, and A. S. Evers, "Anesthesia awareness and the bispectral index," New England Journal of Medicine, vol. 358, no. 11, pp. 1097-1108, 2008, pMID: 18337600

[16] P. Schuller, S. Newell, P. Strickland, and J. Barry, "Response of bispectral index to neuromuscular block in awake volunteers," British Journal of Anaesthesia, vol. 115, pp. 95-103, 2015.

[17] G. Pfurtscheller and F. H. Lopes da Silva, "Event-related EEG/MEG synchronization and desynchronization: basic principles," Clin Neurophysiol, vol. 110, no. 11, pp. 1842-57, Nov 1999.

[18] G. Pfurtscheller, "Induced oscillations in the alpha band: functional meaning," Epilepsia, vol. 44, no. 12, pp. 2-8, 2003.

[19] B. E. Kilavik, M. Zaepffel, A. Brovelli, W. A. MacKay, and A. Riehle, "The ups and downs of beta oscillations in sensorimotor cortex." Exp Neurol, vol. 245, pp. 15-26, Jul 2013.

[20] S. Rimbert, P. Riff, N. Gayraud, D. Schmartz, and L. Bougrain, "Median nerve stimulation based bci: A new approach to detect intraoperative awareness during general anesthesia," Frontiers in $\mathrm{Neu}$ roscience, vol. 13, p. 622, 2019.

[21] Y. Blokland, J. Farquhar, J. Lerou, J. Mourisse, G. J. Scheffer, G.-J. van Geffen, L. Spyrou, and J. Bruhn, "Decoding motor responses from the eeg during altered states of consciousness induced by propofol," Journal of Neural Engineering, vol. 13, no. 2, p. 026014, 2016.

[22] S. Rimbert, R. Al-Chwa, M. Zaepffel, and L. Bougrain, "Electroencephalographic modulations during an open- or closed-eyes motor task," PeerJ, vol. 6, p. e4492, Mar. 2018.

[23] A. Schnitzler, S. Salenius, R. Salmelin, V. Jousmaki, and R. Hari, "Involvement of primary motor cortex in motor imagery: a neuromagnetic study.” Neuroimage, vol. 6, no. 3, pp. 201-208, Oct 1997.

[24] D. Kumbhare, L. Robinson, and R. Buschbacher, Buschbacher's Manual of Nerve Conduction Studies, 3rd Edition. Demos Medical Publishing New york, 2016, ch. Mediane nerve to the abductor pellicis brevis, p. 10.

[25] F. Chung, V. W.S. Chan, and D. Ong, "A post-anesthetic discharge scoring system for home readiness after ambulatory surgery," Journal of clinical anesthesia, vol. 7, pp. 500-6, 101995.

[26] A. Delorme and S. Makeig, "EEGLAB: an open source toolbox for analysis of single-trial eeg dynamics including independent component analysis," Journal of Neuroscience Methods, vol. 134(1), pp. 9-21, 2004.

[27] J.Dien, "Issues in the application of the average reference: review, critiques, and recommendations," Behavior Research Methods, vol. 30, p. 34,1998

[28] B. Blankertz, R. Tomioka, S. Lemm, M. Kawanaba, and K. Müller, "Optimizing spatial filters for robust EEG single-trial analysis," IEEE Signal processing magazine, 2008.

[29] F. Lotte, "A Tutorial on EEG Signal Processing Techniques for Mental State Recognition in Brain-Computer Interfaces," in Guide to Brain-Computer Music Interfacing, E. R. Miranda and J. Caste, Eds. Springer, 2014.

[30] P. Guerci, S. Rimbert, D. Schmartz, M.-R. Losser, L. Bougrain, and C. Meistelman, "Effet du propofol sur les synchronisations/désynchronisations neuronales corticales motrices analysées par une interface cerveau-machine: rapport préliminaire d'une étude prospective chez le volontaire sain," in Congrès SFAR 2019, Paris, France, Sept. 2019.

[31] C. Neuper, M. Wortz, and G. Pfurtscheller, "ERD/ERS patterns reflecting sensorimotor activation and deactivation," Prog Brain Research, vol. 159, pp. 211-222, 2006.

[32] S. Salenius, A. Schnitzler, R. Salmelin, V. Jousmäki, and R. Hari, "Modulation of human cortical rolandic rhythms during natural sensorimotor tasks," NeuroImage, vol. 5, no. 3, pp. 221 - 228, 1997.

[33] E. B. Cooper and J. B. Cooper, "Electrical treatment of coma via the median nerve," in Neurosurgical Re-Engineering of the Damaged Brain and Spinal Cord, Y. Katayama, Ed. Vienna: Springer Vienna, 2003, pp. 7-10.

[34] K. Cassady, A. You, A. Doud, and B. He, "The impact of mind-body awareness training on the early learning of a brain-computer interface," TECHNOLOGY, vol. 02, pp. 254-260, 092014 